\title{
Inverse magnetic catalysis and regularization in the quark-meson model
}

\author{
Jens O. Andersen, ${ }^{a}$ William R. Naylor ${ }^{a}$ and Anders Tranberg $^{b}$ \\ ${ }^{a}$ Department of Physics, Norwegian University of Science and Technology, \\ Høgskoleringen 5, N-7491 Trondheim, Norway \\ ${ }^{b}$ Faculty of Science and Technology, University of Stavanger, \\ N-4036 Stavanger, Norway \\ E-mail: andersen@tf.phys.ntnu.no, william.naylor@ntnu.no, \\ anders.tranberg@uis.no
}

ABSTRACT: Motivated by recent work on inverse magnetic catalysis at finite temperature, we study the quark-meson model using both dimensional regularization and a sharp cutoff. We calculate the critical temperature for the chiral transition as a function of the Yukawa coupling in the mean-field approximation varying the renormalization scale and the value of the ultraviolet cutoff. We show that the results depend sensitively on how one treats the fermionic vacuum fluctuations in the model and in particular on the regulator used. Finally, we explore a $B$-dependent transition temperature for the Polyakov loop potential $T_{0}(B)$ using the functional renormalization group. These results show that even arbitrary freedom in the function $T_{0}(B)$ does not allow for a decreasing chiral transition temperature as a function of $B$. This is in agreement with previous mean-field calculations.

KeYwords: Quark-Gluon Plasma, Chiral Lagrangians, Phase Diagram of QCD, Sigma Models

ARXIV EPRINT: 1410.5247 


\section{Contents}

1 Introduction 1

2 The quark-meson model $\quad 2$

3 Mean-field approximation 3

4 Numerical results $\quad 5$

5 Discussion and conclusion $\quad 9$

\section{Introduction}

In recent years QCD in a strong magnetic field has received considerable attention. This interest has partly been spurred by non-central heavy-ion collisions at the Relativistic Heavy-Ion Collider and the Large Hadron Collider. In these experiments, time-dependent magnetic fields on the order of $|e B| \sim 5 m_{\pi}^{2}$ are created [1-3] and so detailed knowledge of strongly interacting matter in external fields is necessary.

At $T=0$, the response of the QCD vacuum to an external magnetic field is wellknown. Lattice calculations as well as calculations using the Nambu-Jona-Lasinio (NJL) model [4-10], the quark-meson (QM) model [11], Schwinger-Dyson equations for QED [12] and QCD [13], and the Walecka model [14] show that the chiral condensate increases as a function of the external magnetic field $B$. Moreover, even the weakest magnetic fields induce a chiral condensate and thus dynamical chiral symmetry breaking if chiral symmetry is intact at $B=0$.

The fact that the chiral condensate at zero temperature grows as a function of the magnetic field might lead to the expectation that the critical temperature for the chiral transition $\left(T_{c}\right)$ increases as well. Indeed, mean-field calculations employing the NJL model or the Polyakov loop extended NJL (PNJL) [15-17] model as well as the (P)QM model [1823] show that the critical temperature is an increasing function of the magnetic field. This qualitative behavior is independent of the masses of the $\sigma$ and $\pi$ mesons. Additionally, the inclusion of mesonic fluctuations by applying the functional renormalization group (FRG) does not qualitatively change this picture [24-27].

Results from lattice calculations tell a different story. It is seen that the behaviour of $T_{c}$ with $B$ is only increasing at unrealistically large values of the pion masses [28, 29], however with physical pion masses $T_{c}$ is seen to decrease with $B$ [30-34]. A number of groups have begun altering the standard treatment of chiral models to include a mechanism for this inverse magnetic catalysis around $T_{c}[35-41]$. Two such alterations to the PQM model are to allow either the Yukawa coupling, or the transition temperature of the gluonic sector, to 
vary with magnetic field. The former is further motivated by two recent papers utilizing the NJL model that were able to demonstrate inverse magnetic catalysis around $T_{c}$ by varying the four-point coupling [37, 38]. However, in a recent paper by Fraga et al. [35] it was shown that neither of these freedoms were sufficient to obtain inverse magnetic catalysis around $T_{c}$, other than for a limited range of low values of the magnetic field.

Motivated both by the seemingly conflicting results coming from the NJL model, and the extension of the work of Fraga et al. [35] to a functional renormalization group (FRG) treatment we investigate the effects of varying the Yukawa coupling, $g$, within the QM model. We use two different regularization schemes, namely dimensional regularization (DR) and a sharp cutoff. It is seen that simply varying $g$ whilst employing a sharp cutoff gives results that are quantitatively and qualitatively dependent upon the scale of the cutoff, whilst using DR one obtains results that are independent of the renormalization scale. We also investigate varying the transition temperature of the gluonic potential within the PQM model using the FRG and find, in agreement with the prediction of [35], that the FRG does not allow for inverse magnetic catalysis over an extended range of magnetic field values.

It is worth noting that varying the coupling $g$ in an arbitrary fashion amounts to modelling the $B$-dependence of physics beyond the NJL/QM models through an effective coupling $g(B)$. Ideally, such a dependence would be derived directly from QCD, or encoded through some well-motivated higher-dimensional effective operators dependent upon both $g$ and $B$. But as we will see below (see also [35]), even a completely general $g(B)$ seems to be insufficient to provide inverse catalysis, irrespective of its origin. In a sense this is a much stronger conclusion than considering just a single realization of such a dependence. Following [35], we therefore explicitly refrain from specifying the origin of the $B$-dependence og $g$. We will reach a similar conclusion to [35], but also demonstrate that it has certain loop-holes.

The paper is organized as follows. In section 2, we briefly discuss the quark-meson model. In section 3, we calculate the effective potential in the mean-field approximation using different regularizations. In section 4, we present our results for the phase diagram as a function of $g, \Lambda_{\mathrm{UV} / \mathrm{DR}}$ (the cutoff/DR scale) and finally $T_{0}$. In section 5 we discuss the results and briefly summarize our work.

\section{The quark-meson model}

The quark-meson model is a low-energy effective theory for chiral symmetry in QCD. In two-flavor QCD it couples the $O(4)$-symmetric linear sigma model to a massless quark doublet via the Yukawa coupling $g$. The Euclidean Lagrangian in a magnetic field is then given by

$$
\begin{aligned}
\mathcal{L}= & \frac{1}{2}\left[\left(\partial_{\mu} \sigma\right)^{2}+\left(\partial_{\mu} \pi_{0}\right)^{2}\right]+\left(D_{\mu} \pi^{+}\right)^{\dagger} D_{\mu} \pi^{+}+\frac{1}{2} m^{2}\left(\varphi^{\dagger} \varphi\right)+\frac{\lambda}{24}\left(\varphi^{\dagger} \varphi\right)^{2}-h \sigma \\
& +\bar{\psi}\left[\gamma_{\mu} D_{\mu}+g(B)\left(\sigma-\gamma_{5} \boldsymbol{\tau} \cdot \boldsymbol{\pi}\right)\right] \psi
\end{aligned}
$$

where the covariant derivative is $D_{\mu}=\partial_{\mu}-i q_{f} A_{\mu}^{\mathrm{EM}}$, with $q_{f}$ a diagonal matrix of the electric charges of the up and down quarks. $\boldsymbol{\tau}$ are the Pauli matrices, $\varphi^{\dagger}=\left(\sigma, \pi_{0}, \pi_{1}, \pi_{2}\right)$ 
and $\pi^{ \pm}=\frac{1}{\sqrt{2}}\left(\pi_{1} \pm i \pi_{2}\right)$. The fermion field is an isospin doublet,

$$
\psi=\left(\begin{array}{l}
u \\
d
\end{array}\right),
$$

which, as stated, couples to the mesonic sector via the Yukawa coupling $g(B)$, where we have indicated explicitly that this will be allowed to vary with $B$. We make no assumptions as to the manner of this dependence, and simply investigate the available parameter space when any such dependence is allowed (as was done in [35]).

In the absence of external gauge fields the Lagrangian (2.1) is $O(4)$ symmetric if $h=0$ and $O(3)$ symmetric if $h \neq 0$. In the presence of a background Abelian gauge field, the $O(4)$ symmetry is reduced to an $O(2) \times O(2)$ symmetry, because of the different electric charges of the $u$ and $d$ quarks.

Chiral symmetry (or approximate chiral symmetry when $h \neq 0$ ) is broken in the vacuum by a nonzero expectation value $\phi$ for the sigma field. Expanding $\sigma$ around this mean field $\phi$ we define

$$
\sigma=\phi+\tilde{\sigma}
$$

where $\tilde{\sigma}$ is a quantum fluctuating field with vanishing expectation value. The tree-level potential is then

$$
\mathcal{V}_{0}=\frac{1}{2} m^{2} \phi^{2}+\frac{\lambda}{24} \phi^{4}-h \phi
$$

\section{Mean-field approximation}

In the one-loop approximation, one takes into account the Gaussian fluctuations around the mean-field $\phi$. The one-loop effective potential can then be written as a sum of the treelevel term (2.4) and the one-loop contributions from the sigma, the pions, and the quarks. Furthermore, it is a common approximation in the QM model to omit the quantum and thermal fluctuations of the bosons, i.e. treat them at tree level [18, 42].

The one-loop contribution to the effective potential is then given by

$$
\begin{aligned}
\mathcal{V}_{1} & =-\sum_{f} \operatorname{Tr} \log \left[i \gamma_{\mu} D_{\mu}+m_{f}\right] \\
& =\sum_{P_{0}, f, n, s}-\frac{\left|q_{f} B\right|}{2 \pi} \int_{p_{z}} \log \left[P_{0}^{2}+p_{z}^{2}+m_{f}^{2}+\left|q_{f} B\right|(2 n+1-s)\right],
\end{aligned}
$$

where the trace is over Dirac and color indices and in space-time and $m_{u}=m_{d}=g(B) \phi$. Summing over the Matsubara frequencies in eq. (3.1), we find

$\mathcal{V}_{1}=-\sum_{f, n, s} \frac{\left|q_{f} B\right|}{2 \pi} \int_{p_{z}}\left\{\sqrt{p_{z}^{2}+m_{f}^{2}+\left|q_{f} B\right|(2 n+1-s)}-2 T \log \left[1+e^{-\beta \sqrt{p_{z}^{2}+m_{f}^{2}+\left|q_{f} B\right|(2 n+1-s)}}\right]\right\}$. 
The integral over $p_{z}$ for the zero temperature term is divergent and is typically regularized using dimensional regularization in $d=1-2 \epsilon$ dimensions. The sum over Landau levels is then subsequently regulated using $\zeta$-function regularization. The resulting expression is then expanded around $\epsilon=0$ and the poles in $\epsilon$ are removed by renormalization of the parameters in the Lagrangian in the usual way. The details of this calculation can be found in [23]. The result for the renormalized one-loop effective potential reads

$$
\begin{gathered}
\mathcal{V}_{\mathrm{DR}}=\frac{1}{2} m^{2} \phi^{2}+\frac{\lambda}{24} \phi^{4}-h \phi+\frac{N_{c} m_{q}^{4}}{(4 \pi)^{2}} \sum_{f}\left[\log \frac{\Lambda_{\mathrm{DR}}^{2}}{\left|2 q_{f} B\right|}+1\right]-\frac{N_{c}}{2 \pi^{2}} \sum_{f}\left(q_{f} B\right)^{2}\left[\zeta^{(1,0)}\left(-1, x_{f}\right)\right. \\
\left.+\frac{1}{2} x_{f} \log x_{f}\right]-N_{c} \sum_{s, f, k} \frac{\left|q_{f} B\right| T}{\pi^{2}} \int_{0}^{\infty} d p \log \left[1+e^{-\beta \sqrt{p^{2}+M_{q}^{2}}}\right],
\end{gathered}
$$

where $x_{f}=\frac{m_{f}^{2}}{\left|2 q_{f} B\right|}$ and $M_{f}^{2}=\sqrt{m_{f}^{2}+\left|q_{f} B\right|(2 k+1-s)}, \Lambda_{\mathrm{DR}}$ is the renormalization scale associated with the modified minimal subtraction scheme and $\zeta(a, x)$ is the Hurwitz zetafunction.

Although not often employed in renormalizable theories, there is nothing that prevents from using a sharp ultraviolet cutoff $\Lambda_{\mathrm{UV}}$ to regulate the divergent integrals. The effective potential is then

$$
\begin{aligned}
\mathcal{V}_{\text {cut }}= & \frac{1}{2} m^{2} \phi^{2}+\frac{\lambda}{24} \phi^{4}-h \phi+\frac{2 N_{c}}{(4 \pi)^{2}} \sum_{f}\left\{-\Lambda_{\mathrm{UV}} \sqrt{\Lambda_{\mathrm{UV}}^{2}+m_{f}^{2}}\left(2 \Lambda_{\mathrm{UV}}^{2}+m_{f}^{2}\right)\right. \\
& \left.+m_{f}^{4} \log \frac{\Lambda_{\mathrm{UV}}+\sqrt{\Lambda_{\mathrm{UV}}^{2}+m_{f}^{2}}}{m_{f}}-\frac{1}{4} m_{f}^{4}-4\left(q_{f} B\right)^{2}\left[\zeta^{(1,0)}\left(-1, x_{f}\right)-\frac{1}{2}\left(x_{f}^{2}-x_{f}\right) \log x_{f}\right]\right\} \\
& -N_{c} \sum_{s, f, k} \frac{\left|q_{f} B\right| T}{\pi^{2}} \int_{0}^{\infty} d p \log \left[1+e^{-\beta \sqrt{p^{2}+M_{q}^{2}}}\right] .
\end{aligned}
$$

Comparing the two expressions, eqs. (3.3) and (3.4), we see that they have similar structure, other than the presence of an additional term coupling various powers of $m_{f}=g \phi$ and $\Lambda_{\mathrm{UV}}$. Additionally the finite temperature term is independent of the regularization scheme.

The effective potential $\mathcal{V}_{\mathrm{DR}}(\phi)\left(\mathcal{V}_{\text {cut }}(\phi)\right)$ depends upon the parameters $\lambda, m^{2}, g, h$ and $\Lambda_{\mathrm{DR}}\left(\Lambda_{\mathrm{UV}}\right)$. As we will explore the dependence of the transition temperatures on $\Lambda_{\mathrm{DR}}$ and $\Lambda_{\mathrm{UV}}$, these are left as completely 'free'. Without explicit symmetry breaking the pions are true Goldstone bosons, i.e. we need not fix $m_{\pi}$ as it is automatically zero. At nonzero pion mass we adjust $h$ to set $m_{\pi}$. The pion decay constant, $f_{\pi}$, and sigma mass $m_{\sigma}$ in the vacuum, $T=B=0$, are fixed by tuning $\lambda$ and $m^{2}$. All these must be tuned for every different value of $\Lambda_{\mathrm{DR}}$ or $\Lambda_{\mathrm{UV}}$ and of course they will be different for the different regularization schemes. We use the values $f_{\pi}=93 \mathrm{MeV}$ and $m_{\sigma}=530 \mathrm{MeV}$ throughout this paper, and at the physical point $m_{\pi}=139 \mathrm{MeV}$. Finally the Yukawa coupling, $g=g(B)$, at $B=0$ is set to 3.2258 such that the constituent quark mass is $g \phi=300 \mathrm{MeV}$. However at finite $B$ and $T$ we will allow this to vary whilst holding $m^{2}$ and $\lambda$ fixed. 

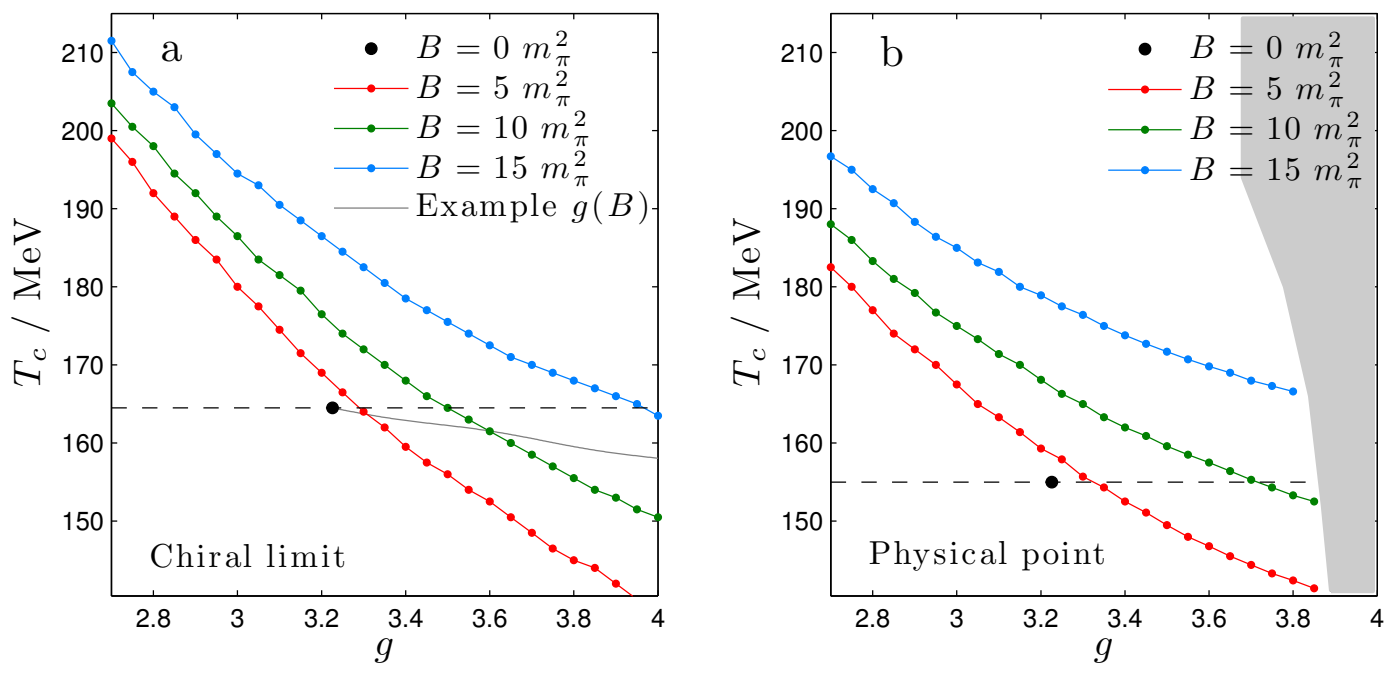

Figure 1. Chiral transition temperature as a function of possible value of the Yukawa coupling for the chiral limit (1a) and the physical point (1b). The plots show that a function $g(B)$ starting at $g=3.2258$, $\bullet$, can give inverse magnetic catalysis up to around $10 m_{\pi}^{2}$ at the physical point. Beyond this the theory breaks down (grey region). See text for details.

\section{Numerical results}

Our initial motivation was to use the FRG to see if the conclusions in ref. [35] would be altered when including mesonic fluctuations. It turns out that FRG seems to open for the possibility of inverse magnetic catalysis. However, the flow equation involves integration of momenta $k$ from a sharp ultraviolet cutoff $\Lambda_{\mathrm{FRG}}$ down to $k=0$ and the naive use of this cutoff proves problematic.

Following ref. [35], in figure 1, we plot the critical temperature for the chiral transition as a function of the Yukawa coupling $g(B)$ for various values of $B$. Figure 1a gives the results in the chiral limit, while figure $1 \mathrm{~b}$ is at the physical point. The curves are obtained using the dimensionally regulated mean-field result, eq. (3.3), with $\Lambda_{\mathrm{DR}}=182 \mathrm{MeV}$, as was used in [23]. The results shown in figure 1 are in approximate agreement with those of Fraga et al. [35]. At the physical point (1b) we see that the critical temperature becomes undefined at high $B$ and $g(B)$, as given by the grey region. We return to this point shortly.

Figure 1 is understood as follows: at $B=0$ the constituent quark mass fixes the Yukawa coupling, and thus the chiral transition temperature is fixed to be $165 \mathrm{MeV}$ ( $155 \mathrm{MeV}$ at the physical point), as is given by $\bullet$. The dashed black line is simply a visual guide to distinguish catalysis from inverse catalysis. Moving to finite magnetic field the value of the Yukawa coupling as a function of $B$ and $T$ is not known, thus we allow for any possible dependence. Any particular function $g(B)$ is a curve beginning at $\bullet$ and successively intersecting the various contours of increasing $B$. One such function $g(B)$ is given by the grey line in figure 1a. If the functional dependence is given by

$$
g(B)=g(0)\left[1+a\left(B / m_{\pi}^{2}\right)^{b}\right]
$$



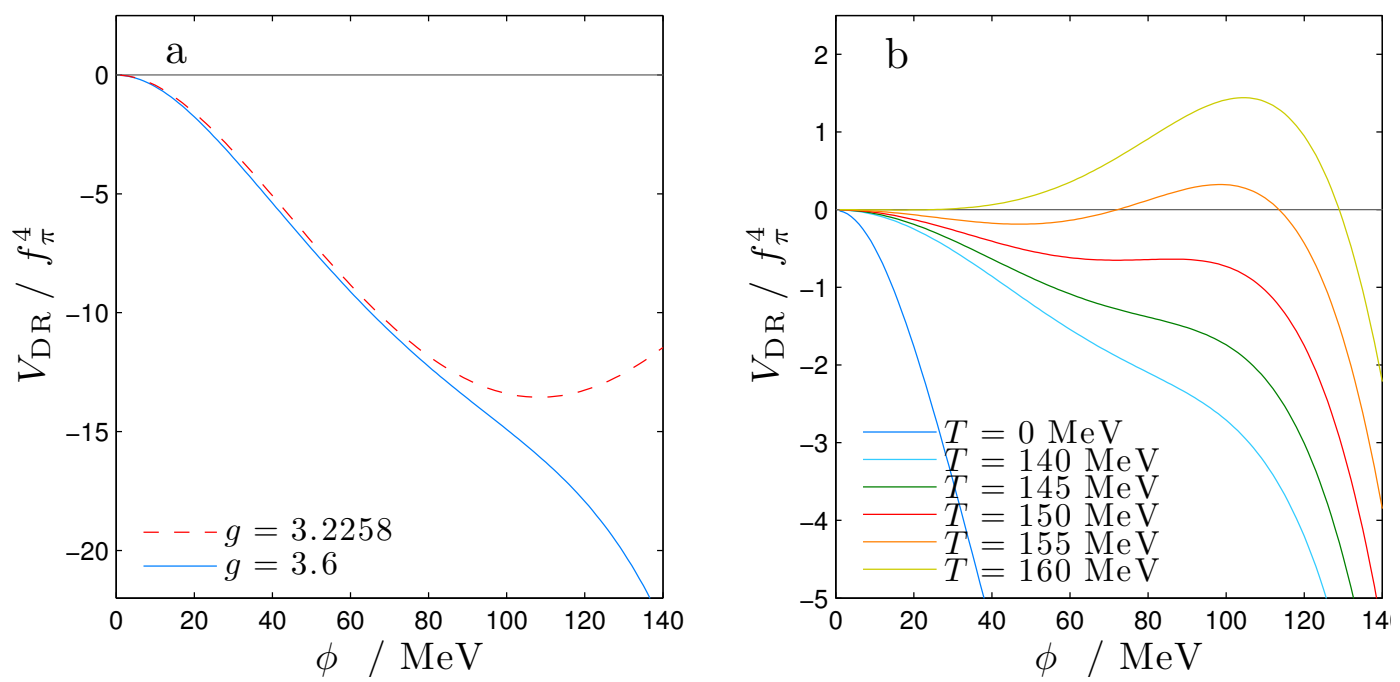

Figure 2. 2a gives the mean-field potential in the chiral limit for $g=3.2258$ and 3.6 at $T=0$. We see at $g=3.6$ that the theory has an unstable vacuum. $2 \mathrm{~b}$ is the potential with $g=3.6$ for increasing $T$. It is seen that the potential develops a local minimum at positive, finite $\phi$ before the transition temperature, which lies at $161.5 \mathrm{MeV}$. In both plots $B=10 \mathrm{~m}_{\pi}^{2}$.

then this grey line corresponds approximately to $a=0.0008$ and $b=2.2$. Here $g$ increases with $B$ in such a way as to give inverse magnetic catalysis up to at least $12 m_{\pi}^{2}$. An even simpler function is a line moving vertically upwards from $\bullet$. This corresponds to the standard case, where one assumes the Yukawa coupling is independent of $B$ and $T$ i.e. $a=b=0$. In this case, of course, we find $T_{c}$, increasing with $B$, i.e. magnetic catalysis.

After inspection of figure 1a it seems quite possible to create a function $g(B)$ such that we have inverse magnetic catalysis over a large range of magnetic field strength. However the complete picture is more complex as is shown at the physical point, given in figure 1b. Firstly, the change in the definition of the critical temperature (from a second order transition, to a cross over with a pseudocritical temperature) flattens the curves of constant $B$ such that a greater change in $g$ is required for the same change in $B$ and the total range of $B$ values over which one could have inverse catalysis is reduced (for example the blue $B=15 m_{\pi}^{2}$ curve may never cross the dashed line even if it could be continued to infinitely high $g$ ). More problematic than this, at large $g$ (given by the grey region), the theory breaks down. We now explain this with the help of figure 2.

As is well known, at very large values of the field $\phi$ the mean-field potential becomes unbounded from below, due to the log term in the zero temperature expression (eq. (3.3) for the DR scheme). This term is proportional to the fourth power of the quark mass, so it is greatly influenced by varying the Yukawa coupling. This is evident from figure 2a, where we see at $T=0 \mathrm{MeV}, B=10 \mathrm{~m}_{\pi}^{2}$ that changing $g$ from 3.2258 to 3.6 , the local minimum disappears altogether giving us unbounded (unphysical) results, indeed for $g>3.35$ this is the case. Thus if $g$ is only a function of $B$ then we may not vary it higher than 3.35. However, in figure $2 \mathrm{~b}$ we now show the change in the potential with $T$ with $B=10 \mathrm{~m}_{\pi}^{2}$, 


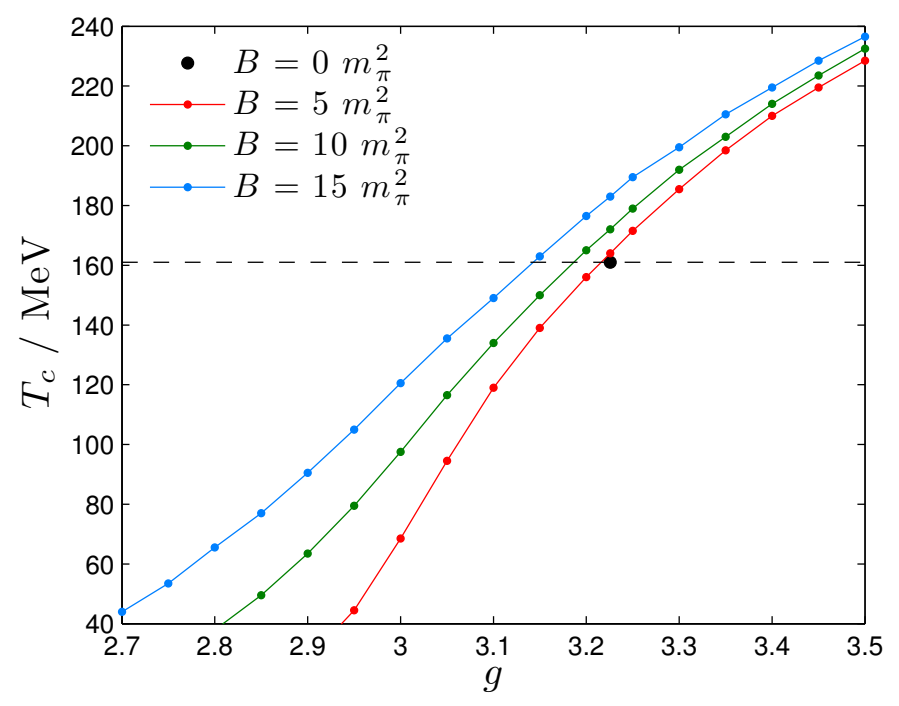

Figure 3. $T_{c}$ plotted against $g$ using a sharp cutoff of $800 \mathrm{MeV}$. It is seen that a decreasing function $g(B)$ could give inverse magnatic catalysis, however, as noted in the text, this result depends heavily upon the value of the cutoff.

$g=3.6$ and in the chiral limit. As $T$ increases, we first develop a local minimum, like we have in the zero $T, g=3.2258$ case, and then the potential undergoes the usual chiral phase transition. Because the transition temperature in the chiral limit essentially involves investigating the potential around $\phi=0$ we are able to define the transition temperature even for very large values of $g$ and $B$. But as $g$ is pushed higher and higher, the region where we have a local minimum becomes smaller, both in $T$ and $\phi$. For this reason at finite $B$ we will allow $g$ to be a function of both $B$ and $T$ such that we have the maximum flexibility in $g$ with which to obtain inverse magnetic catalysis. At the physical point the definition of the pseudocritical temperature involves investigation of a finite value of the field $\phi$, in our case where it is equal to half the zero temperature value (but note this is not changed when investigating the inflection point). In this case even moderate values of $g$ and $B$ give unphysical results, as is given by the grey region in figure $1 \mathrm{~b}$. This is the primary reason that disallows inverse magnetic catalysis even allowing $g$ to be a function of both $B$ and $T$.

We now turn out attention to the QM model with a sharp cutoff, choosing a value of $800 \mathrm{MeV}$, as was used in our previous calculations using FRG [25, 26]. The plot corresponding to the previous figure 1 is given in figure 3 . These results are qualitatively the same as those we found using the FRG. The observed behaviour is reversed as compared with the dimensionally regulated result, most obviously $T_{c}$ is an increasing function of $g$ for any fixed $B$ is, while it was a decreasing function in the dimensionally regulated theory. In addition we see that it is possible to choose a function $g(B, T)$ that gives inverse magnetic catalysis. Both as the curves become steeper as we move from 3.2258 to approximately 3 (the region of interest here), but also because $g$ must decrease to obtain inverse magnetic catalysis, thus avoiding the problems of an unbounded potential seen above. However we 


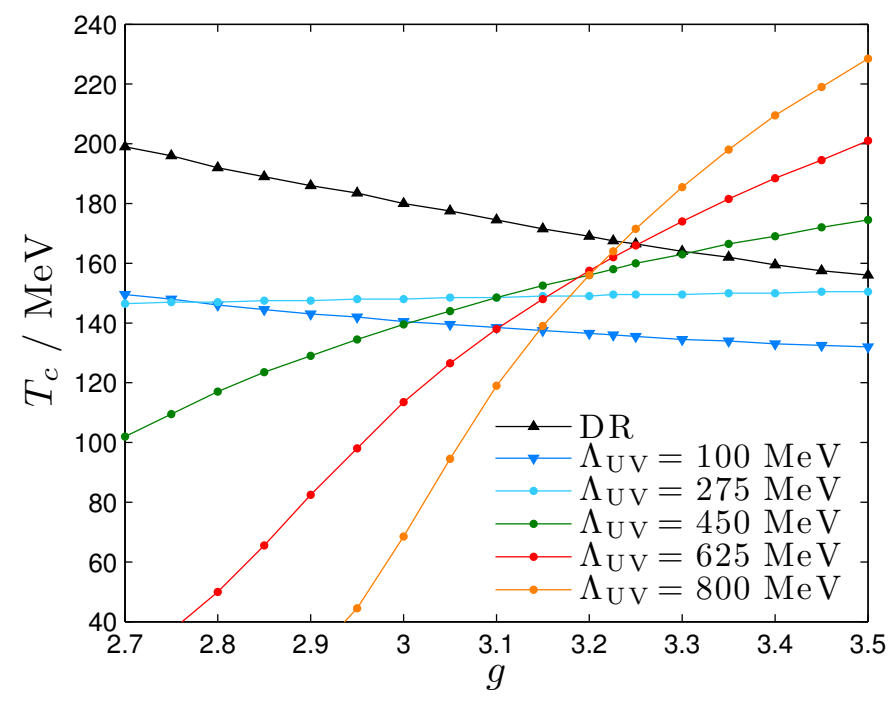

Figure 4. $T_{c}$ with $g(B)$ for various values of $U_{\mathrm{DR}}$ and $U_{\mathrm{UV}}$ for both regularization schemes. For the dimensionally regularized theory only a single curve (black 'up' triangles) is given as all values of the renormalization scale between 100 and $800 \mathrm{MeV}$ have $T_{c}$ within $\pm 2 \mathrm{MeV}$ of the curve given, which is calculated using $\Lambda=182 \mathrm{MeV}$. In the sharp cutoff theory with $\Lambda=100 \mathrm{MeV}$ (blue 'down' triangles) the phase transition is of first order, all other transition are second order. The plot shows the heavy dependence of the cutoff theory on the value of the cutoff.

stress that this result is heavily dependent upon the cutoff used and thus this conclusion should not be used out of context. We now turn our attention to this cutoff dependence.

In figure 4 we plot $T_{c}$ for only a single value of the magnetic field, instead varying the cutoff. The parameter fixing we discussed in section 3 is done for each different value of $\Lambda_{\mathrm{DR}}$ and $\Lambda_{\mathrm{UV}}$, thus at $g=3.2258, f_{\pi}, m_{\sigma}, m_{\pi}$ and $m_{q}$ are equal for each curve. We plot only a single value of $\Lambda_{\mathrm{DR}}$ for the theory using DR as the results are within $\pm 2 \mathrm{MeV}$ for all cutoff values between 100 and $800 \mathrm{MeV}$. For both regularization schemes the finite $T$ terms are exactly the same, and the zero $T$ components are also very similar with exception that the sharp cutoff theory adds a term of the form $-\Lambda_{\mathrm{UV}} \sqrt{\Lambda_{\mathrm{UV}}^{2}+m_{f}^{2}}\left(2 \Lambda_{\mathrm{UV}}^{2}+m_{f}^{2}\right)$. Figure 4 shows that using a large cutoff this term begins to dominate the behaviour as we increase $g$, lowering the potential and thus increasing $T_{c}$.

It has been suggested that the backreaction of the quarks to the gluons plays a primary role in inverse magnetic catalysis [33], thus a natural first step towards inverse magnetic catalysis in Polyakov loop coupled models would be via tuning the gluonic potential. In [35] this was done at mean-field level, where they concluded that it was not possible to obtain inverse magnetic catalysis by simply varying the gluonic transition temperature, $T_{0}$. We show that this result remains unchanged with the inclusion of mesonic fluctuations in figure 5, which is calculated using the FRG. We do not introduce the full machinery of the FRG, instead referring the reader to [26]. The methods are the same as in that paper, other than that we rerun the calculation for varying values of $T_{0}$, which is a free parameter in the model. For the physical observables $f_{\pi}, m_{f}, m_{\pi}$ and $m_{\sigma}$, the values are the same as 


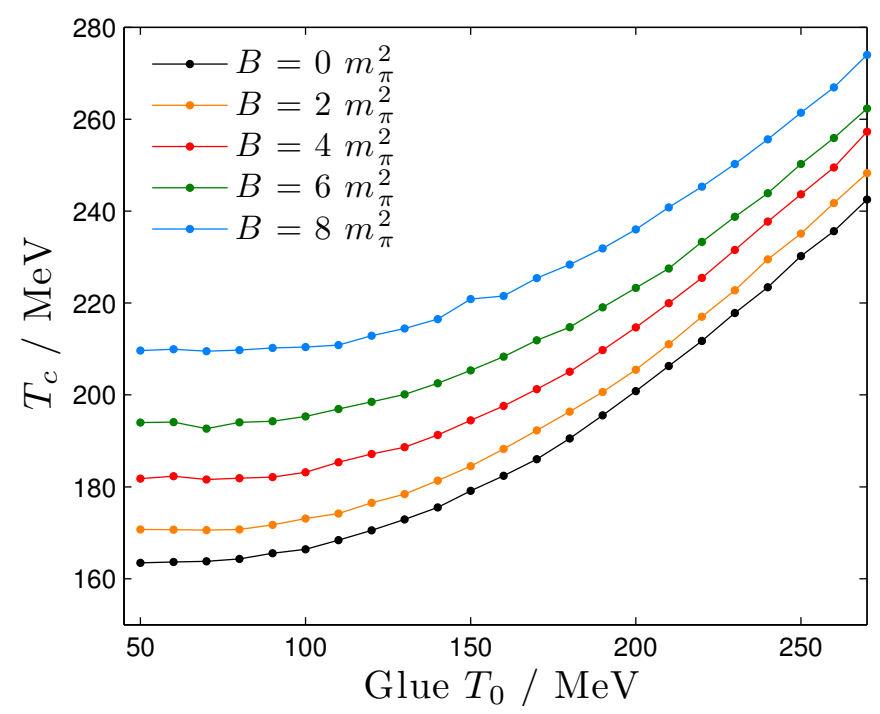

Figure 5. $T_{c}$ with varying $T_{0}$ for various values of $B$. Note that the scaling in $B$ is more gradual than in previous plots. We see that it is not possible to have inverse magnetic catalysis given any possible function $T_{0}(B)$ if we also require that $T_{c}(B=0) \sim T_{d}(B=0)$.

we used in the physical point, mean-field calculations and we have $\Lambda_{\mathrm{FRG}}=800 \mathrm{MeV}$. The figure can be read in the same way as figure 1 , but with $T_{0}$ in the place of $g$. At $B=0, T_{0}$ is usually taken as $\sim 210 \mathrm{MeV}$ with two flavours of quarks. This value also ensures that $T_{c}$ is approximately equal to the deconfinement transition temperature, $T_{d}$. Using this as the zero- $B$ starting point we see that the curves are simply too flat (and become increasingly so as we decrease $T_{0}$ ) to allow for inverse magnetic catalysis up to magnetic fields over $B \sim 4 m_{\pi}^{2}$. Thus we find in agreement with the prediction of [35] that using the FRG, there is no possible function $T_{0}(B)$ which could give inverse magnetic catalysis.

\section{Discussion and conclusion}

In the quark-meson model, one chooses a regularization prescription and fixes the associated scale $\left(\Lambda=\Lambda_{\mathrm{DR}}, \Lambda_{\mathrm{UV}}, \Lambda_{\mathrm{FRG}}\right)$ and then sets $m^{2}, \lambda, h$ and $g$ to obtain the correct values of the particle masses and pion decay constant in the vacuum. In doing so the model's dependence upon $\Lambda$ is essentially cancelled out. The model is useful because we may then vary external parameters without introducing strong $\Lambda$ dependence and thus investigate physics outside of the vacuum. But in varying $g$ there is no guarantee that the model will be $\Lambda$ independent, and as figure 4 shows, when using a cutoff this is not the case. The real problem here, in terms of modelling inverse magnetic catalysis, is not only that it is not possible to generate meaningful results in the mean-field approximation using a sharp cutoff, but that it is not possible to do so using the FRG, as it suffers from exactly the same problems.

Usually it is the case that the inclusion of mesonic fluctuations has some quantitative effect upon the chiral transition temperature but that the results remain qualitatively the 
same. However we have seen from lattice results that inverse magnetic catalysis is dependent upon the pion mass. But usually in model calculations only the mesonic fluctuations are dependent upon the pion mass, thus indicating their importance. Moreover varying the pion mass amounts to varying $m^{2}$ and $\lambda$ in eq. (3.3) or (3.4). But as there is no coupling between $g$ and either of these variables, varying $m_{\pi}$ simply shifts all of the curves in figure 1 either up or down, something we have checked explicitly. Thus to fully reproduce the lattice results at mean-field by varying $g$ (if it was even possible) one would need $g\left(B, T, m_{\pi}\right)$.

We agree with the basic result of Fraga et al. [35], that within the QM model it is not possible to reproduce lattice results by simply utilizing $g(B)$, even if we allow complete freedom in this functional dependence. However, as we use different regularization the reason for this is very different. Moreover we find within our own results that the transition temperature as a function of $g$ depends in detail of how one treats the vacuum fluctuations. It is not only a question of whether to include them or not, as in the case of the order of the transition, but it also depends upon the exact implementation of the regularization scheme. Allowing $g$ to run with $B$ acknowledges that there exists physics not captured by the quark-meson model yet vital in mapping out the chiral phase diagram. But this physics must be incorperated in such a way that reliable computations can still be made. This is not the case when simply varying $g$ using a sharp cutoff and only approximately true in dimensional regularization.

We have refrained from explicitly stating where the effective $B$-dependence comes from. In full renormalizable QCD the $B$-dependence can not depend on the regularization, and the expectation is therefore that any effective theory should share this feature. The way forward seems to be to augment the PNJL or PQM model with a selection of additional operators and perform a consistent renormalization of the quantum theory. In particular any such operators would provide additional counterterms to cancel out residual cutoff dependence through some additional renormalization condition.

\section{Acknowledgments}

AT is supported by a Villum Foundation Young Investigator Grant.

Open Access. This article is distributed under the terms of the Creative Commons Attribution License (CC-BY 4.0), which permits any use, distribution and reproduction in any medium, provided the original author(s) and source are credited.

\section{References}

[1] D.E. Kharzeev, L.D. McLerran and H.J. Warringa, The Effects of topological charge change in heavy ion collisions: 'Event by event P and CP-violation', Nucl. Phys. A 803 (2008) 227 [arXiv: 0711.0950] [INSPIRE].

[2] V. Skokov, A.Y. Illarionov and V. Toneev, Estimate of the magnetic field strength in heavy-ion collisions, Int. J. Mod. Phys. A 24 (2009) 5925 [arXiv:0907.1396] [INSPIRE].

[3] A. Bzdak and V. Skokov, Event-by-event fluctuations of magnetic and electric fields in heavy ion collisions, Phys. Lett. B 710 (2012) 171 [arXiv:1111.1949] [INSPIRE]. 
[4] S.P. Klevansky and R.H. Lemmer, Chiral symmetry restoration in the Nambu-Jona-Lasinio model with a constant electromagnetic field, Phys. Rev. D 39 (1989) 3478 [InSPIRE].

[5] D. Ebert, K.G. Klimenko, M.A. Vdovichenko and A.S. Vshivtsev, Magnetic oscillations in dense cold quark matter with four fermion interactions, Phys. Rev. D 61 (2000) 025005 [hep-ph/9905253] [INSPIRE].

[6] K.G. Klimenko, Three-dimensional Gross-Neveu model at nonzero temperature and in an external magnetic field, Z. Phys. C 54 (1992) 323 [inSPIRE].

[7] V.P. Gusynin, V.A. Miransky and I.A. Shovkovy, Catalysis of dynamical flavor symmetry breaking by a magnetic field in (2+1)-dimensions, Phys. Rev. Lett. 73 (1994) 3499 [Erratum ibid. 76 (1996) 1005] [hep-ph/9405262] [INSPIRE].

[8] V.P. Gusynin, V.A. Miransky and I.A. Shovkovy, Dimensional reduction and catalysis of dynamical symmetry breaking by a magnetic field, Nucl. Phys. B 462 (1996) 249 [hep-ph/9509320] [INSPIRE].

[9] C.N. Leung, Y.J. Ng and A.W. Ackley, Schwinger-Dyson equation approach to chiral symmetry breaking in an external magnetic field, Phys. Rev. D 54 (1996) 4181 [hep-th/9512114] [INSPIRE].

[10] D.S. Lee, C.N. Leung and Y.J. Ng, Chiral symmetry breaking in a uniform external magnetic field, Phys. Rev. D 55 (1997) 6504 [hep-th/9701172] [INSPIRE].

[11] E.J. Ferrer and V. de la Incera, Magnetic catalysis in the presence of scalar fields, Phys. Lett. B 481 (2000) 287 [hep-ph/0004113] [INSPIRE].

[12] C.N. Leung and S.-Y. Wang, Gauge independent approach to chiral symmetry breaking in a strong magnetic field, Nucl. Phys. B 747 (2006) 266 [hep-ph/0510066] [INSPIRE].

[13] N. Mueller, J.A. Bonnet and C.S. Fischer, Dynamical quark mass generation in a strong external magnetic field, Phys. Rev. D 89 (2014) 094023 [arXiv:1401.1647] [INSPIRE].

[14] A. Haber, F. Preis and A. Schmitt, Magnetic catalysis in nuclear matter, Phys. Rev. D 90 (2014) 125036 [arXiv:1409.0425] [inSPIRE].

[15] R. Gatto and M. Ruggieri, Dressed Polyakov loop and phase diagram of hot quark matter under magnetic field, Phys. Rev. D 82 (2010) 054027 [arXiv: 1007.0790] [INSPIRE].

[16] R. Gatto and M. Ruggieri, Deconfinement and Chiral Symmetry Restoration in a Strong Magnetic Background, Phys. Rev. D 83 (2011) 034016 [arXiv: 1012.1291] [InSPIRE].

[17] K. Kashiwa, Entanglement between chiral and deconfinement transitions under strong uniform magnetic background field, Phys. Rev. D 83 (2011) 117901 [arXiv:1104.5167] [INSPIRE].

[18] E.S. Fraga and A.J. Mizher, Chiral transition in a strong magnetic background, Phys. Rev. D 78 (2008) 025016 [arXiv:0804.1452] [INSPIRE].

[19] A.J. Mizher, M.N. Chernodub and E.S. Fraga, Phase diagram of hot QCD in an external magnetic field: possible splitting of deconfinement and chiral transitions, Phys. Rev. D 82 (2010) 105016 [arXiv: 1004.2712] [INSPIRE].

[20] D.C. Duarte, R.L.S. Farias and R.O. Ramos, Optimized perturbation theory for charged scalar fields at finite temperature and in an external magnetic field, Phys. Rev. D 84 (2011) 083525 [arXiv: 1108.4428] [INSPIRE]. 
[21] M. Ruggieri, M. Tachibana and V. Greco, Renormalized vs. Nonrenormalized Chiral Transition in a Magnetic Background, JHEP 07 (2013) 165 [arXiv:1305.0137] [INSPIRE].

[22] M. Ruggieri, L. Oliva, P. Castorina, R. Gatto and V. Greco, Critical Endpoint and Inverse Magnetic Catalysis for Finite Temperature and Density Quark Matter in a Magnetic Background, Phys. Lett. B 734 (2014) 255 [arXiv:1402.0737] [INSPIRE].

[23] J.O. Andersen and R. Khan, Chiral transition in a magnetic field and at finite baryon density, Phys. Rev. D 85 (2012) 065026 [arXiv:1105.1290] [INSPIRE].

[24] V. Skokov, Phase diagram in an external magnetic field beyond a mean-field approximation, Phys. Rev. D 85 (2012) 034026 [arXiv: 1112.5137] [INSPIRE].

[25] J.O. Andersen and A. Tranberg, The Chiral transition in a magnetic background: Finite density effects and the functional renormalization group, JHEP 08 (2012) 002 [arXiv: 1204.3360] [INSPIRE].

[26] J.O. Andersen, W.R. Naylor and A. Tranberg, Chiral and deconfinement transitions in a magnetic background using the functional renormalization group with the Polyakov loop, JHEP 04 (2014) 187 [arXiv:1311.2093] [INSPIRE].

[27] K. Kamikado and T. Kanazawa, Chiral dynamics in a magnetic field from the functional renormalization group, JHEP 03 (2014) 009 [arXiv: 1312.3124] [INSPIRE].

[28] M. D'Elia, S. Mukherjee and F. Sanfilippo, QCD Phase Transition in a Strong Magnetic Background, Phys. Rev. D 82 (2010) 051501 [arXiv:1005.5365] [InSPIRE].

[29] M. D'Elia and F. Negro, Chiral Properties of Strong Interactions in a Magnetic Background, Phys. Rev. D 83 (2011) 114028 [arXiv:1103.2080] [InSPIRE].

[30] G. Endrodi, Z. Fodor, S.D. Katz and K.K. Szabo, The QCD phase diagram at nonzero quark density, JHEP 04 (2011) 001 [arXiv: 1102.1356] [INSPIRE].

[31] G.S. Bali, F. Bruckmann, G. Endrodi, Z. Fodor, S.D. Katz et al., QCD quark condensate in external magnetic fields, Phys. Rev. D 86 (2012) 071502 [arXiv:1206.4205] [INSPIRE].

[32] G.S. Bali, F. Bruckmann, G. Endrodi, Z. Fodor, S.D. Katz et al., The QCD phase diagram for external magnetic fields, JHEP 02 (2012) 044 [arXiv:1111.4956] [INSPIRE].

[33] F. Bruckmann, G. Endrodi and T.G. Kovacs, Inverse magnetic catalysis and the Polyakov loop, JHEP 04 (2013) 112 [arXiv: 1303.3972] [INSPIRE].

[34] G.S. Bali, F. Bruckmann, G. Endrodi, F. Gruber and A. Schaefer, Magnetic field-induced gluonic (inverse) catalysis and pressure (an)isotropy in QCD, JHEP 04 (2013) 130 [arXiv: 1303.1328] [INSPIRE].

[35] E.S. Fraga, B.W. Mintz and J. Schaffner-Bielich, A search for inverse magnetic catalysis in thermal quark-meson models, Phys. Lett. B 731 (2014) 154 [arXiv:1311.3964] [INSPIRE].

[36] M. Ferreira, P. Costa, D.P. Menezes, C. Providência and N. Scoccola, Deconfinement and chiral restoration within the SU(3) Polyakov-Nambu-Jona-Lasinio and entangled Polyakov-Nambu-Jona-Lasinio models in an external magnetic field, Phys. Rev. D 89 (2014) 016002 [arXiv: 1305.4751] [INSPIRE].

[37] R.L.S. Farias, K.P. Gomes, G.I. Krein and M.B. Pinto, Importance of asymptotic freedom for the pseudocritical temperature in magnetized quark matter, Phys. Rev. C 90 (2014) 025203 [arXiv: 1404.3931] [INSPIRE]. 
[38] M. Ferreira, P. Costa, O. Lourenço, T. Frederico and C. Providência, Inverse magnetic catalysis in the (2+1)-flavor Nambu-Jona-Lasinio and Polyakov-Nambu-Jona-Lasinio models, Phys. Rev. D 89 (2014) 116011 [arXiv:1404.5577] [InSPIRE].

[39] E.J. Ferrer, V. de la Incera and X.J. Wen, Quark Antiscreening at Strong Magnetic Field and Inverse Magnetic Catalysis, arXiv:1407.3503 [INSPIRE].

[40] A. Ayala, M. Loewe, A.J. Mizher and R. Zamora, Inverse magnetic catalysis for the chiral transition induced by thermo-magnetic effects on the coupling constant, Phys. Rev. D 90 (2014) 036001 [arXiv: 1406.3885] [INSPIRE].

[41] A. Ayala, M. Loewe and R. Zamora, Inverse magnetic catalysis in the linear $\sigma$-model with quarks, Phys. Rev. D 91 (2015) 016002 [arXiv: 1406.7408] [INSPIRE].

[42] O. Scavenius, A. Mócsy, I.N. Mishustin and D.H. Rischke, Chiral phase transition within effective models with constituent quarks, Phys. Rev. C 64 (2001) 045202 [nucl-th/0007030] [INSPIRE]. 